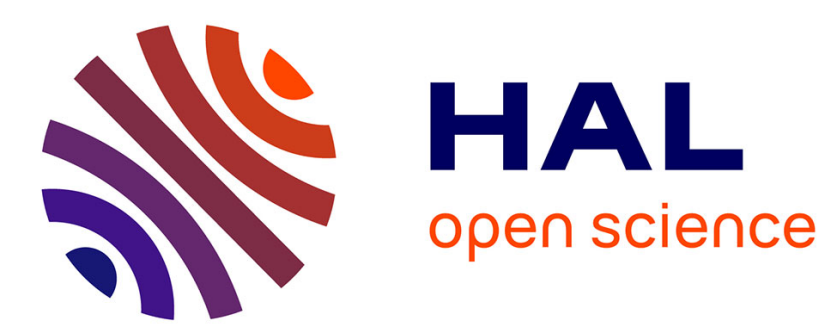

\title{
Calculated Ventilation and Effort Distribution as a Measure Of Respiratory Disease and Heliox Effectiveness
}

Nicolas Pozin, Spyridon Montesantos, Ira Katz, Marine Pichelin, Céline

Grandmont, Irene Vignon-Clementel

\section{- To cite this version:}

Nicolas Pozin, Spyridon Montesantos, Ira Katz, Marine Pichelin, Céline Grandmont, et al.. Calculated Ventilation and Effort Distribution as a Measure Of Respiratory Disease and Heliox Effectiveness. Journal of Biomechanics, 2017, 60C, pp.100-109. 10.1016/j.jbiomech.2017.06.009 . hal-01468861

\author{
HAL Id: hal-01468861 \\ https://hal.science/hal-01468861
}

Submitted on 15 Feb 2017

HAL is a multi-disciplinary open access archive for the deposit and dissemination of scientific research documents, whether they are published or not. The documents may come from teaching and research institutions in France or abroad, or from public or private research centers.
L'archive ouverte pluridisciplinaire HAL, est destinée au dépôt et à la diffusion de documents scientifiques de niveau recherche, publiés ou non, émanant des établissements d'enseignement et de recherche français ou étrangers, des laboratoires publics ou privés. 


\title{
Calculated Ventilation and Effort Distribution as a Measure Of Respiratory Disease and Heliox Effectiveness
}

\author{
N. Pozin ${ }^{1,2,3}$, S. Montesantos ${ }^{3}$, I. Katz ${ }^{3,4}$, M. Pichelin ${ }^{3}$, C. Grandmont ${ }^{1,20^{*}}$, I. Vignon-Clementel $\mathrm{I}^{1,20^{*}}$ \\ ${ }^{1}$ INRIA Paris, 2 Rue Simone Iff, 75012 Paris, France \\ ${ }^{2}$ Sorbonne Universités, UPMC Univ. Paris 6, Laboratoire Jacques-Louis Lions, 75252 Paris, France \\ ${ }^{3}$ Medical R\&D, WBL Healthcare, Air Liquide Santé International, 1 Chemin de la Porte des Loges, 78350 Les \\ Loges-en-Josas, France \\ ${ }^{4}$ Department of Mechanical Engineering, Lafayette College, Easton, PA, 18042, USA \\ • shared last authorship \\ *corresponding author: \\ celine.grandmont@inria.fr, irene.vignon-clementel@inria.fr \\ INRIA Paris, 2 Rue Simone Iff, 75015 Paris, France \\ $+33(0) 1.80 .49 .42 .67$
}

Keywords : asthma, $\mathrm{HeO}_{2}$, lung, mathematical modeling, stochastic respiratory tree

Original article

3485 words 


\begin{abstract}
In spite of numerous clinical studies, there is no consensus on the benefit Heliox mixtures can bring to asthmatic patients in terms of work of breathing and ventilation distribution. In this article we use a 3D finite element mathematical model of the lung to study the impact of asthma on effort and ventilation distribution along with the effect of Heliox compared to air. Lung surface displacement fields extracted from computed tomography medical images are used to prescribe realistic boundary conditions to the model. Asthma is simulated by imposing bronchoconstrictions to some airways of the tracheo-bronchial tree based on statistical laws deduced from the literature. This study illuminates potential mechanisms for patient responsiveness to Heliox when affected by obstructive pulmonary diseases. Responsiveness appears to be function of the pathology severity, as well as its distal position in the tracheo-bronchial tree and geometrical position within the lung.
\end{abstract}

\title{
1. Introduction
}

The lung is a complex multiscale system. Furthermore, many in-vivo measurements, especially as a function of position within the lung parenchyma, are difficult or impossible to perform. Thus, mathematical models can provide a unique understanding of physical phenomena associated with fundamental physiology and respiratory disease. In (Berger et al., 2015) and (Pozin et al., 2016) 3D lung models were developed and used to study the impact of airway bronchoconstrictions on ventilation and pressure distributions within the parenchyma. In this paper we perform in silico asthma experiments and focus on the variety of response to an helium-oxygen mixture (Heliox or $\left.\mathrm{HeO}_{2}\right)$.

Helium is a low density inert gas. Heliox has been studied as a means to ease breathing and improve ventilation in obstructive lung diseases since the 1930s (Barach, 1935). However, consistent benefit demonstrated in randomized clinical trials has yet to follow thus preventing widespread use. For 
example, a Cochrane review (Rodrigo et al., 2002) concluded that current evidence is insufficient to support the use of $\mathrm{HeO}_{2}$ mixtures in the treatment of acute exacerbations of COPD and future controlled trials are advocated. Recently, a large trial (16 intensive care units, 6 countries) found that $\mathrm{HeO}_{2}$ improves respiratory acidosis, encephalopathy and respiratory rate more quickly than $\mathrm{Air} / \mathrm{O}_{2}$ for COPD patients but does not prevent NIV failure(Jolliet et al., 2016). In (Häussermann et al., 2015) healthy controls, moderate to severe asthmatic and COPD patients inhaled $\mathrm{HeO}_{2}$ during exercise. The impact on lung function and metabolic cost showed no statistical difference compared to air even though in each group there were some responders and non-responders. The authors emphasized the need to better understand the influence of disease and disease severity on responsiveness to Heliox. There are few modeling attempts to study the effect of breathing $\mathrm{HeO}_{2}$ compared to Air. In (Katz et al., 2011) a resistance model accounting for gas density effect on pressure drops in a bifurcation is proposed and applied to Heliox mixtures. In (Katz et al., 2014) the impact of breathing $\mathrm{HeO}_{2}$ on particle deposition is studied, CFD, in-vivo imaging and bench study suggest deeper deposition compared to air. In this paper, we use a numerical modeling approach to give insights on why, for a given pathology, some patients respond to $\mathrm{HeO}_{2}$ and some others do not. In the following, responsiveness to Heliox is judged based on how the gas helps to decrease work of breathing and reduce ventilation defaults compared to air. We use a lung finite element model in which the tracheo-bronchial tree and the surrounding tissues are coupled (Pozin et al., 2016). To simulate obstructive diseases, some airways can be constricted. Depending on which and how many airways are narrowed, we evaluate responsiveness to $\mathrm{HeO}_{2}$.

\section{Model and methods}

\subsection{Lung structure}

The lung consists of a porous media, called the parenchyma, supplied with gas through a dyadic branching structure, the tracheo-bronchial tree (see Figure 1); each level of branching is called a 
generation (beginning with 0 for the trachea). Each branch is assumed to be a rigid cylinder. Viscous and inertial effects induce energy dissipations through the airways. We use the nonlinear Pedley branch resistance model (Pedley, Schroter and Sudlow, 1970) to account for pressure drops within a bifurcating pipe:

$$
R_{\text {ped }}=\left\{\begin{array}{lc}
\gamma\left(R e \frac{2 r}{L}\right)^{\frac{1}{2}} R_{\text {pois }}, \quad \gamma\left(\operatorname{Re} \frac{2 r}{L}\right)^{\frac{1}{2}}>1, \\
R_{\text {pois }}, \quad \gamma\left(\operatorname{Re} \frac{2 r}{L}\right)^{\frac{1}{2}}<1,
\end{array}\right.
$$

where $\gamma=0.327, L$ and $r$ are respectively the branch length and radius, $R e$ is the Reynolds number defined by $R e=\frac{2 \rho|q|}{\mu_{f} \pi r}$ with $\rho$ the fluid density, $\mu_{f}$ its dynamic viscosity, $q$ the flow rate through the branch and the laminar Poiseuille resistance is $R_{\text {pois }}=\frac{8 \mu_{f} L}{\pi r^{4}}$. The term $\gamma\left(\operatorname{Re} \frac{2 r}{L}\right)^{\frac{1}{2}}$ is associated with inertial losses, sensitive to the fluid (gas) density. In the following, the extra-thoracic component is neglected.

The parenchyma is assumed to be an isotropic elastic medium occupying a domain $\Omega$. Following (Cazeaux and Grandmont, 2012) we treat it as a homogenized material characterized by effective macroscopic mechanical parameters. As tidal breathing is slow we choose to neglect tissue viscosity. The related linearized stress tensor is given by:

$$
\sigma_{m a t}(\varepsilon(\boldsymbol{u}))=2 \mu \varepsilon(\boldsymbol{u})+\lambda \operatorname{tr}(\varepsilon(\boldsymbol{u})) I
$$

where $\lambda$ and $\mu$ are the effective Lamé parameters, $\boldsymbol{u}$ is the parenchyma displacement defined in the reference state domain $\Omega$, and $\varepsilon$ the linear strain tensor defined by $\varepsilon(\boldsymbol{u})=\frac{1}{2}\left(\nabla \boldsymbol{u}+{ }^{T} \nabla \boldsymbol{u}\right)$.

Note that (1) has been designed for a given angle and at inspiration only. In fact as shown in 
(MY.Kang, J.Hwang and JW.Lee, 2011), it can be used independently of the angle. Besides, we choose to use it at expiration as well. As in tidal breathing displacements are moderate, a large deformation non linear constitutive relation may be more appropriate for other respiratory regimes such as spirometry and mechanical ventilation.

\subsection{Tree-parenchyma coupled model}

The tree and parenchyma are coupled through the approach introduced in (Pozin et al., 2016). Neglecting gravity, the momentum equation of the system is:

$$
\operatorname{div}\left(\sigma_{\text {mat }}+\sigma_{\text {coupling }}\right)=\rho_{\text {par }} \frac{\partial^{2} \boldsymbol{u}}{\partial t^{2}}, \quad \text { in } \Omega,
$$

where $\rho_{\text {par }}$ is the parenchyma density and $\sigma_{\text {coupling }}$ is the tree-parenchyma coupling term given by

$$
\sigma_{\text {coupling }}=-p_{\text {tree }} I \text {. }
$$

Let $\Omega_{i}$ be the sub-region of $\Omega$ that is fed in gas by the $i^{\text {th }}$ tree exit; $p_{\text {tree }}$ is the piecewise constant function defined by

$$
p_{\text {tree }}=P_{\text {trachea }}-P_{i} \text { in } \Omega_{i}
$$

where $P_{\text {trachea }}$ and $P_{i}$ are the pressure at the trachea entrance and at the $i^{\text {th }}$ tree exit, respectively. The action of the tree on the parenchyma is similar to an apparent pressure (see Figure 1), the greater the pressure drop along the path to an exit, the greater the resistance to the fed region's volume variations.

To complete the problem, the lung surface motion is prescribed as the boundary condition to Equation (2) (see Section 2.3). The same global ventilation scenario is thus imposed, whatever the 
diseased state and inhaled gas.

Then to evaluate the benefit of Heliox compared to air we propose local 3D quantities as well as global average markers to account for respiratory effort. We define a pressure distribution within the parenchyma called local effective pressure $p_{\text {eff }}$. It consists in an elastic component $p_{\text {elastic }}$ associated with the elastic recoil and a component $p_{\text {tree }}$ associated with the tree. It is given at time $t$ by:

$$
p_{\text {eff }}(\boldsymbol{x}, t)=p_{\text {elastic }}(\boldsymbol{x}, t)+p_{\text {tree }}(\boldsymbol{x}, t)=K \operatorname{div}(\boldsymbol{u}(\boldsymbol{x}, t))+p_{\text {tree }}(\boldsymbol{x}, t) \text { in } \Omega
$$

where $K$ is the parenchyma bulk modulus that is a function of the Lamé parameters. In this definition shear stress and inertia are neglected. We also define the global average quantities

$$
P_{\text {elastic }}=\int_{\Omega}\left|p_{\text {elastic }}\right|
$$

and

$$
P_{\text {tree }}=\int_{\Omega}\left|p_{\text {tree }}\right|
$$

which can respectively be seen as a measure of the instantaneous effort needed to deform an elastic material along the displacement field $\boldsymbol{u}$, and the effort needed to induce the corresponding flow distribution through the tree, respectively.

The work $W$ of $p_{\text {eff }}$ over a time period $T$ is defined as:

$$
W=\int_{0}^{T} \int_{\Omega}\left[\left(\operatorname{Kdiv}(\boldsymbol{u}(\boldsymbol{x}, t))+p_{\text {tree }}(\boldsymbol{x}, t)\right) \operatorname{div}(\boldsymbol{v}(\boldsymbol{x}, t))\right]
$$


where $v$ is the parenchyma velocity field defined by $v=\frac{\partial u}{\partial t}$.

\subsection{Patient-specific structural elements}

A high resolution computed tomography (HRCT) image, at mean lung volume (MLV), for a single subject (Greenblatt et al., 2014) was the basis to build a patient-specific tree and the parenchyma geometry. The segmented upper airways and lung envelope were the initial conditions used to build a lobar space-filling tree following the methodology described in (Montesantos et al., 2016). From the lung envelope a 3D mesh is built using Meshlab (Meshlab - developped with the support of 3DCoForm project - meshlab.sourceforge.net, n.d.) and Gmsh (Geuzaine and Remacle, 2009). Using Deformetrica (Durrleman et al., 2014) we register a surface displacement field to map the mean to the total lung capacity volume HRCT-based envelopes given in (Greenblatt et al., 2014). To generate a tidal look-alike breathing profile, this motion is bounded and sinusoidal dynamics are imposed. The resulting displacement is prescribed as boundary condition (BC) of the model.

\subsection{Modeling disease of the tracheo-bronchial tree}

Asthma and COPD affect the tracheo-bronchial lung tree structure. Inflammation can induce airway constrictions (Montaudon et al., 2009) (Kotaru et al., 1985) up to closure (Montesantos et al., 2013)(Aikawa et al., 1992). While some studies argue these pathologies mostly occur in small airways (Burgel, 2011) (Carr et al., 1998), autopsies on patients who died from status asthmaticus (Dunnill, 1960) (Aikawa et al., 1992) and CT scans of diseased subjects have shown upper airways can also be affected (Montesantos et al., 2013). Little quantitative data is provided in the literature about which particular airways are affected in asthma and to what extent. Some studies show slight but frequent airway narrowing. In (Aikawa et al., 1992) measured lumen area exhibited radius decreases of about $10 \%$ on average compared to controls. In (Montaudon et al., 2009) CT measurements showed reductions of 10 to $25 \%$. In addition to those small constrictions, (Carr et al., 1998) demonstrates the presence of airway closures in asthmatic patients. In (Montesantos et al., 2013) segmented images of 
asthmatic patients indicate that severe stenoses can completely occlude airways. Non-published data from this study give the number of closures per segmented tree.

To simulate a bronchoconstriction with diameter reduction ratio $r$ (simply referred as "ratio" in the following) we divide the diameter of the affected branch by $r$ ranging from 1 (healthy) to 10 (airway closure). Furthermore, based on the literature data we simulate an asthma attack with constriction ratios generated according to a log-normal law $\mathfrak{L}$ with average $\log (1.2)$, standard deviation 0.3 and within bounds $[1 ; 3]$. We also impose $n_{\text {closure }}$ severe constrictions (plugs), $n_{\text {closure }}$ being an integer randomly selected with uniform law in the interval $\llbracket 1 ; 40 \rrbracket$. Plugs are applied at random positions and with constriction ratios randomly selected with the uniform law in the interval $[4 ; 10]$. In order to compare the effect of severe and moderate obstructions, we can impose $n_{\text {closure }}$ severe plugs only, or, alternatively, only divide all the airways diameters by ratios computed following $\mathfrak{L}$. Resulting trees will be qualified respectively as "plug only" and "plug free".

\section{Results and discussion}

In this section lung ventilation simulation results and breathing effort are provided. Local and global differences between healthy and pathological cases are explored. We first simulate a single bronchoconstriction on an upper airway and study the effect of breathing $\mathrm{HeO}_{2}$ compared to air on both ventilation and $W$. We then analyze how responsiveness to $\mathrm{HeO}_{2}$ evolves depending on which region of the lung is affected and when smaller airways are constricted. After having described the physical principles on simplified bronchoconstrictions, asthma attacks are introduced following Section 2.4 .

Simulations were performed on a left lung geometry model. A convergence analysis led to a 51495 tetrahedral mesh. Mechanical properties are assumed to be homogeneous and are set within the ranges provided in (Epstein and Ligas, 1990): Young's modulus $E=1256 \mathrm{~Pa}$ and Poisson ratio $v=0.4$ where $E$ and $v$ are linked to the Lamé parameters by $\lambda=\frac{v E}{(1+v)(1-2 v)}$ and $\mu=\frac{E}{2(1+v)}$. 
Parenchyma density is $\rho=100 \mathrm{~kg} \cdot \mathrm{m}^{-3}$. The tree contains ten generations leading to 477 exits. Each exit airway resistance includes an additive term to account for the remaining sub-tree resistance. The lobe nomenclature is: $L U=L$ eft Upper, $L L=L e f t$ Lower. Branches feeding lobes $L U$ and $L L$ are called the "LU branch" and "LL branch", respectively. Each region "Reg" contains two sub-regions "RegR" and "RegL" served by a "RegR branch" and a "RegL branch" respectively. If not specified, the breathing period is $4 \mathrm{~s}$, the reference state lung volume is $1.04 \mathrm{~L}$ and maximum volume expansion is $0.23 \mathrm{~L}$. Air density and viscosity at $37^{\circ} \mathrm{C}$ are respectively $1.125 \mathrm{~kg} \cdot \mathrm{m}^{-3}$ and $18.9510^{-6} \mathrm{~Pa} . \mathrm{s}$, the $\mathrm{HeO}_{2}$ mixture contains $78 \% \mathrm{He} / 22 \% \mathrm{O}_{2}$, density is $0.434 \mathrm{~kg} \cdot \mathrm{m}^{-3}$ and viscosity is $22.5610^{-6} \mathrm{~Pa}$.s. As stated in Section 2.3, a displacement BC is applied. The overall lung volume evolution is thus imposed and, if not specified, identical for all the simulations.

\subsection{Impact of a bronchoconstriction}

In this section a bronchoconstriction with varying ratios is imposed on the LU branch. Lobar volume expansions are plotted for both air and $\mathrm{HeO}_{2}$ in Figure 2.A and 2.B. First a healthy case is simulated (Figure 2.A), volumes evolve in phase and lobar ventilation is not affected by the inhaled gas, inertial effects are negligible. In Figure 2.B are the results for a bronchoconstriction with ratio 7 applied to the LU branch. Lobe LU expands less and does not expel all the gas it contains, gas trapping is typical of asthma and COPD (Laurent et al., 2000). Ventilation of the diseased region is improved when breathing $\mathrm{HeO}_{2}$ compared to air. Indeed, velocity in the constricted airway, and hence inertial effects, are increased because of the diameter reduction. This effect is all the more important when the gas density is high (see (1)) so resistance to Air is higher than resistance to $\mathrm{HeO}_{2}$. Finally, in order to breathe a given amount of gas, $\mathrm{HeO}_{2}$ flow results in lower pressure drops than air making it easier to breathe. For a given lung surface displacement, gas distributes preferentially in regions where it goes more easily so lobe LL is more ventilated. In Figure 2.C and Figure 2.D, $P_{\text {elastic }}$ and $P_{\text {tree }}$, are plotted. In the healthy case (Figure 2.C), $P_{\text {elastic }}$ is much greater than $P_{\text {tree }}$. Regarding ventilation effort, efforts are negligibly changed when inhaling $\mathrm{HeO}_{2}$. In Figure 2.D a bronchoconstriction with ratio 5 is 
applied to the LU branch. This ratio was chosen for representation because it emphasizes most pressure differences between healthy and constricted configurations while a factor 7 exhibited a higher gap for ventilation. Component $P_{\text {tree }}$ is no longer negligible compared to $P_{\text {elastic }}$. Breathing $\mathrm{HeO}_{2}$ is clearly easier.

Figure 3.A shows contour plots of the relative volume expansion (see 2.2) on an interior surface of the lung at time 1.68s for both gases in a healthy case and for a bronchoconstriction with ratio 7 applied on the LU branch. We first note that volume expansion is heterogeneous and greater at the base than the apex. Indeed the diaphragm is more active than the ribs during tidal breathing. As already observed in Figure 2.A, the ventilation distribution is not affected by the gas in the healthy model. In the pathological situation, the $\mathrm{LU}$ lobe is less expanded, and breathing $\mathrm{HeO}_{2}$ mitigates the ventilation heterogeneity. In Figure 3.B are plots of $p_{\text {eff }}$, as defined by (4), at time $1 \mathrm{~s}$. The local effort in the diseased region is reduced with $\mathrm{HeO}_{2}$, consistent with Figure 2.B.

Depending on the pathologic severity, response to $\mathrm{HeO}_{2}$ varies. Figure 4.A shows the evolution of the LU lobe volume amplitude as a function of the bronchoconstriction applied to the LU branch; for both gases. For every ratio the $\mathrm{LU}$ lobe is better ventilated with $\mathrm{HeO}_{2}$ than with air. The more the diameter reduction, the more the inertial effects and the higher the ventilation difference between both gases, up to $27 \%$ for tested cases. In Figure 4.B, $W$ over one respiration cycle is studied. Up to a ratio 7, it appears easier to breathe $\mathrm{HeO}_{2}$ than air. For higher ratios the difference between the two gases is inversed. As a function of the pressure drop along the tree, $W$ is a balance of resistances and flows. As the constriction gets more severe, inertial effects are increased so the resistance to air flow increases more than the resistance to $\mathrm{HeO}_{2}$. Concurrently to the resistance ratio decrease, the $\mathrm{HeO}_{2}$ to air flow rate ratio increases. Thus, after an interval of constrictions where $W$ is lower for $\mathrm{HeO}_{2}, \mathrm{~s}$ it becomes comparable for the two gases and eventually $W$ is higher for $\mathrm{HeO}_{2}$. Note that the total volume variation is imposed, whatever the effort it takes to reach; so for strong ratios the system may exhibit non-physiological efforts. 
As a conclusion, responsiveness to $\mathrm{HeO}_{2}$ depends on the pathologic severity. A low density gas helps reducing $W$ if the pathology is not too severe and improves ventilation of diseased regions in all studied cases. However, this model does not include a resistance through the extra-thoracic region where inertial losses may be sufficient to make $\mathrm{HeO}_{2}$ consistently easier to breath than air (Sandeau et al., 2010).

\subsection{Impact of the regional position}

In this section we study how the regional position of the pathology within the lung influences the responsiveness to $\mathrm{HeO}_{2}$. To do so we compare the ventilation in three lung regions LURL, LULR, LULL for varying reduction ratios. Those regions have the same reference state volume but different expansions over the breathing cycle (Figure 5).

In Figure 6 we see that for high reduction ratios the resulting flow rates are too low to induce inertial effects so $\mathrm{HeO}_{2}$ /air differences become insignificant. Note that in the absence of inertial effects pressure drops are purely viscous; since air viscosity is lower, the relative difference becomes negative though absolute values are close. For intermediate ratios, the difference between both gases increases with regional expansion because inertial effects are higher.

As a conclusion responsiveness to $\mathrm{HeO}_{2}$ is highest for intermediate pathology severity and depends on the position within the lung.

\subsection{Impact of the generational position}

As noted before, responsiveness to $\mathrm{HeO}_{2}$ is higher for higher flows. From proximal to tidal regions, flows decrease as they distribute within the tree. Depending on which generations are affected by the pathology, responsiveness to $\mathrm{HeO}_{2}$ may be different. In this section we apply a bronchoconstriction on all the airways of the LU lobe belonging to generation $n$, for $n \in \llbracket 2 ; 9 \rrbracket$. For each "diseased generation", ventilations obtained with both gases are compared for varying ratios. 
Figure 7 shows two different regimes. When proximal generations ( 2 and 3 ) are affected, responsiveness to $\mathrm{HeO}_{2}$ increases with the bronchoconstriction ratio. For more distal generations responsiveness increases with constriction severity, but then decreases as flows become too slow to induce inertial effects. Starting at generation $8, \mathrm{HeO}_{2}$ brings no benefit compared to air. As a conclusion, in case of bronchoconstriction, $\mathrm{HeO}_{2}$ seems to be beneficial when upper airways are affected, but this is not the case as the pathology reaches more distal areas, here after generation 8 .

\subsection{Asthma attack simulation}

In previous sections one or more localized bronchoconstrictions with diameter reduction ratios varying from 2 to 10 were simulated. Here we simulate an asthma crisis with a combination of severe obstructions and lower radius changes, as described in 2.4. Following this process fifty "asthma attack trees" are built. To compare the effect of severe and moderate obstructions, "plug free" and "plug only" related trees are simulated (see 2.4).

During a crisis patients may panic and experience breathing difficulties and tend to breathe faster with lower volumes, even though slow breathing is advised (Thomas and Bruton, 2014). To study the effect of breathing $\mathrm{HeO}_{2}$ and the impact of respiration frequency we perform the simulations listed in table 1. Results are presented in Figure 8 and Figure 9.

In the healthy case, $\mathrm{HeO}_{2}$ does not ease breathing. For asthmatic attack models, there is high breathing effort variability. Heliox brings a $24 \%$ improvement on average and up to $30 \%$. Note that $W$ is increased in average by a factor 6.7 from healthy cases to asthma when breathing air but by a factor 5.2 when breathing Heliox. Variability is reduced when only moderate bronchoconstrictions are simulated but Heliox does perform better than air in both "plug free" and "plug only" configurations. Note that on average breathing is harder with numerous moderate plugs than with a few severe ones, but variability is much higher in the latter case. This is consistent with the results in previous sections.

In the 2 second breathing period case (so-called "T2"), breathing is faster and shortened. In this configuration, flows are increased, inertial effects are more important and Heliox reduces $W$ over 
one period by $23 \%$. A similar reduction is observed in the 4 second breathing period " $\mathrm{T} 4$ " case, but in terms of energy spent per unit of time, Heliox is two times more beneficial in the fast breathing case. It also appears that breathing air two times more slowly brings more profit regarding effort reduction than breathing $\mathrm{HeO}_{2}$ at the same pace.

A Kolmogorov-Smirnov test was performed and showed no statistical differences between the gases. That is, on average there is no statistical benefit but there are responders to $\mathrm{HeO}_{2}$ that do benefit.

\section{Conclusion}

This study provides insights on respiratory diseases in the form of bronchoconstrictions and their effect on ventilation distribution and effort together with Heliox responsiveness. We focus on why some patients might respond to $\mathrm{HeO}_{2}$ and others do not. Low density mixtures can be beneficial compared to air when flows within the tree exhibit marked inertial effects. This is especially true when constricted airways are in proximal generations. Simulating localized constrictions provides a physical understanding of the effects leveraging responsiveness to $\mathrm{HeO}_{2}$. There is a range of constriction ratios, where $\mathrm{HeO}_{2}$ is most beneficial, and this range depends on the regional and generational positions. Asthma was then simulated based on the geometrical variability reported in the literature. The work of breathing is multiplied in average by a factor 6.7 from health to asthma when breathing air, and by a factor 5.2 when breathing heliox. During an asthma crisis, breathing can be volume restricted and frequency increased; Heliox mixtures prove to be more helpful in that case. Slow breathing can be a more efficient way than $\mathrm{HeO}_{2}$ to decrease efforts and improve ventilation distribution. These findings may help diagnose potential responders based on their tracheo-bronchial morphometry.

\section{Acknowledgements}

This work was supported by GRANT ANR-11-TECS-006). NP was funded by ANRT. The authors gratefully acknowledge Mr. Fabien Raphel for his great support with the felisce code (FELISCE, n.d.) 


\section{Conflict of Interest}

Airliquide is a producer of Heliox. There are no known conflicts of interest related to this publication.

\section{References}

Aikawa, T., Shimura, S., Sasaki, H., Ebina, M. and Takishima, T. (1992) 'Marked goblet cell hyperplasia with mucus accumulation in the airways of patients who died of severe acute asthma attack', Chest, no. 101(4):916-21.

B.Maury (2013) The Respiratory System in Equations, Springer.

Barach, A. (1935) 'The use of helium as a new therapeutic gas', Anesth Analg, no. 14:210-213.

Berger, L., Bordas, R., Burrowes, K., Grau, V., Tavener, S. and Kay, D. (2015) 'A poroelastic model coupled to a fluid network with applications in lung modelling', Int J Numer Method Biomed Eng, vol. 32(1):e02731.

Burgel, P. (2011) 'The role of small airways in obstructive airway diseases', Eur Respir Rev, no. 20(119):23-33.

Carr, D., Hibon, S., Rubens, M. and Chung, K. (1998) 'Peripheral airways obstruction on highresolution computed tomography in chronic severe asthma', Respir Med, no. 92(3):448-53.

Cazeaux, P. and Grandmont, C. (2012) 'Homogenization of a multiscale viscoelastic model with nonlocal damping, application to the human lungs', Mathematical Models and Methods in Applied Sciences, vol. 25(06):1125-1177.

Dunnill, M. (1960) 'The pathology of asthma, with special reference to changes in the bronchial mucosa', J Clin Pathol, no. 13(1): 27-33.

Durrleman, S., Prastawa, M., Charon, N., Korenberg, J., Joshi, S., Gerig, G. and Trouvé, A. (2014) 'Morphometry of anatomical shape complexes with dense deformations and sparse parameters', Neurolmage, vol. 101:35-49.

Epstein, M. and Ligas, J. (1990) Respiratory biomechanics.

FELISCE, [Online], Available: https://gforge.inria.fr/projects/felisce/.

Geuzaine, C. and Remacle, J. (2009) 'Gmsh: a three-dimensional finite element mesh generator with built-in pre- and post-processing', Int. J. Numer. Meth. Engng, vol. 79(11):1309-1331.

Greenblatt, E., Winkler, T., Harris, R., Kelly, V., Kone, M. and J, V. (2014) 'Analysis of threedimensional aerosol deposition in pharmacologically relevant terms: beyound black or white ROIs', $J$ Aerosol Med Pulm Drig Deliv, no. 28(2):116-29, Available: 10.1089/jamp.2013.1120.

Häussermann, S., Schulze, A., Katz, I., Martin, A., Herpich, C., Hunger, T. and Texereau, J. (2015) 'Effects of a helium/oxygen mixture on individuals' lung function and metabolic cost during 
submaximal exercise for participants with obstructive lung diseases', International Journal of COPD, no. 2015: 10(1).

Ismail, M., Comerford, A. and Wall, W. (2013) 'Coupled and reduced dimensional modeling of respiratoy mechanics during spontaneous breathing', Int. J. Numer. Meth. Biomed. Engng, no. 10.1002/cnm.2577, Available: 10.1002/cnm.2577.

Jolliet, P., Ouanes-Besbes, L., Abroug, F., Ben Khelil, J., Besbes, M., Garneo, A., Arnal, J., Daviaud, F., Chich, J., Lortat-Jacob, B., Diehl, J., Lerolle, N., Mercat, A., Razazi, K., Brun-Buisson, C., Durand-Zaleski, I., Texereau, J. and Brochard, L. (2016) 'A multicenter randomized trial assessing the efficacy of helium/oxygen in severe exacerbations of chronic obstructive pulmonary disease', Am J Respir Crit Care Med, no. 10.1164/rccm.201601-00830C.

Kass, J. and Terregino, C. (1999) 'The effect of heliox in acute severe asthma: a randomized controlled trial', Chest, vol. 116(2):296-300.

Katz, I., Martin, A., Muller, P., Terzibachi, K., Feng, C., Caillibotte, G., Sandeau, J. and Texereau, J. (2011) 'The ventilation distribution of helium-oxygen mixtures and the role of inertial losses in the presence of heterogeneous airway obstructions', J Biomech, no. 44(6):1137-43, Available: 44(6):1137-43.

Katz, I., Pichelin, M., Montesantos, S., Majoral, C., Martin, A., Conway, J., Fleming, J., Venegas, J., Greenblatt, E. and Caillibotte, G. (2014) 'Using helium-oxygen to improve regional deposition of inhaled particles: mechanical principles', J Aerosol Med Pulm Drug Deliv, no. 27(2):71-80.

Kotaru, C., Coreno, A., Skowronski, M., Muswick, G., Gilkeson, R. and McFadden, E.J. (1985) 'Morphometric changes after thermal and methacholine bronchoprovocations', J Appl Physiol, no. 98(3):1028-36.

Laurent, F., Latrabe, V., Raherison, C., Marthan, R. and Tunon-de-Lara, J. (2000) 'Functional significance of air trapping detected in moderate asthma', Eur Radiol, no. 10(9):1404-10.

Meshlab-developped with the support of 3D-CoForm project - meshlab.sourceforge.net.

Montaudon, M., Lederlin, M., Reich, S., Begueret, H., Tunon-de-Lara, J., Marthan, R., Berger, P. and Laurent, F. (2009) 'Bronchial measurements in patients with asthma: comparison of quantitative thinsection CT findings with those in healthy subjects and correlation with pathologic findings', Radiology, vol. 253(3):844-53.

Montesantos, S., Katz, I., Fleming, J., Majoral, K., Pichelin, M., Dubau, C., Piednoir, B., Conway, J., Texereau, J. and Caillibotte, J. (2013) 'Airway morphology from high resolution computed tomography in healthy subjects and patients with moderate persistent asthma', The anatomical record, no. 296:852-866.

Montesantos, S., Katz, I., Pichelin, M. and Caillibote, G. (2016) 'The creation and statistical evaluation of a deterministic model of the human bronchial tree from HRCT images', PLOS one, Available: 10.1370/journal.pone.0168026. 
MY.Kang, J.Hwang and JW.Lee (2011) 'Effect of geometric variations on pressure loss for a model bifurcation of the human lung airway', J.Biomech, no. 44(6):1196-9.

Pedley, T., Schroter, R. and Sudlow, M. (1970) 'Energy losses and pressure drops in models of human airways', Respir Physiol, no. 10.1016/0034-5687(70)90093-9, Available: 9(3):371-86.

Pozin, N., Montesantos, S., Katz, I., Pichelin, M., Vignon-Clementel, I. and Grandmont, C. (2016) 'A tree-parenchyma coupled model for lung ventilation simulation', Int J Numer Method.

Rodrigo, G., Pollack, C., Rodrigo, C. and Rowe, B. (2002) 'Heliox for treatment of exacerbations of chronic obstructive pulmonary disease', Cochrane Database Syst Rev, no. 2002;(2):CD003571.

Sandeau, J., Katz, I., Fodil, R., Apiou-Sbirlea, G., Caillibotte, G. and Isabey, D. (2010) 'CFD simulation of particle deposition in a reconstructed human oral extrathoracic airway for air and helium-oxygen mixtures', Journal of aerosol science, no. 41.3(2010):281-294.

Thomas, M. and Bruton, A. (2014) 'Breathing exercises for asthma', Breathe, no. 10: 312-322.

\section{Figure captions}


Figure 1: The parenchyma occupies a domain $\Omega$, fed in gas through a dyadic tree structure. This domain is subdivided into non-intersecting regions $\Omega_{\mathrm{i}}$, each of them being supplied through the $\mathrm{i}^{\text {th }}$ tree exit. Green arrows represent the apparent pressure applied on terminal region $\Omega_{1}$ due to the coupling with the tree.

Figure 2: Lobar ventilation ( $\mathrm{A}$ and $\mathrm{B}$ ) and $P_{\text {elastic }}$ (eqn. (5)) and $P_{\text {tree }}$ (eqn.(6)) efforts ( $C$ and $D$ ) through a sinusoidal respiration cycle for both air and $\mathrm{HeO}_{2}$ in a healthy situation $(A$ and $C$ ) and when a bronchoconstriction is applied to LU branch (B: ratio 7, D: ratio 5). See the text for the ratio choices.

Figure 3: Left lung slice from basis to apex representing: (A) the relative volume expansion $\boldsymbol{d i v}(\boldsymbol{u})$ in a healthy case and when a bronchoconstriction with ratio 7 is applied to LU branch, for both Air and $\mathrm{HeO}_{2}$ at time $1.68 \mathrm{~s}$, (B) the local pressure peff defined by (4) in a healthy case and when a bronchoconstriction with ratio 5 is applied to $\mathrm{LU}$ branch, for both Air and $\mathrm{HeO}_{2}$ at time 1 s. See the text for the ratio choices.

Figure 4: Bronchoconstriction applied on LU branch with varying ratios, evolution of: (A) LU lobe volume maximum amplitude over a breathing period for both Air and $\mathrm{HeO}_{2}$, and the relative difference between both gases, (B) $\boldsymbol{W}$ (defined by eqn. (7)) over a breathing period for both Air and $\mathrm{HeO}_{2}$

Figure 5: Three comparable regions belonging to the left lung are picked (A). They have the same reference state volume (B) but different volume expansions over the breathing cycle (C).

Figure 6: Ventilation in three lung regions. Volume amplitude with Air and $\mathrm{HeO}_{2}$ for varying ratios (A) and relative difference between both gases for each region (B).....

Figure 7: Bronchoconstrictions are applied to all the airways of a given generation belonging to LU lobe. Each curve corresponds to a different "diseased generation" and shows the maximum inhaled volume relative difference between both gases (VmaxHeO2 - VmaxAir VmaxAir ) for varying ratios.

Figure 8: average and standard deviations of the $\boldsymbol{W}$ over a respiration cycle in different configurations: one healthy patient breathing air and $\mathrm{HeO}_{2}$, fifty asthmatic configurations ith bronchonconstrictions simulated according to 2.4, fifty patients with only diffuse moderate bronchoconstrictions (plug free) and fifty patients with only severe bronchoconstrictions (plug only).

Figure 9: average and standard variations of the $\boldsymbol{W}$ over a respiration cycle in different configurations: one healthy patient breathing air and $\mathrm{HeO}_{2}$, fifty asthmatic patients with bronchoconstrictions simulated according to 2.4 breathing air and $\mathrm{HeO}_{2}$ with time period $4 \mathrm{~s}$ and volume expansions $0.23 \mathrm{~L}(\mathrm{~T} 4)$, the same fifty asthmatic patients breathing air and $\mathrm{HeO}_{2}$ with time period $2 \mathrm{~s}$ and volume expansions $0.16 \mathrm{~L}(\mathrm{~T} 4)$, the same fifty asthmatic patients breathing air with time period $8 \mathrm{~s}$ and volume expansions $0.23 \mathrm{~L}(\mathrm{~T} 8)$.

\section{Figures and tables}




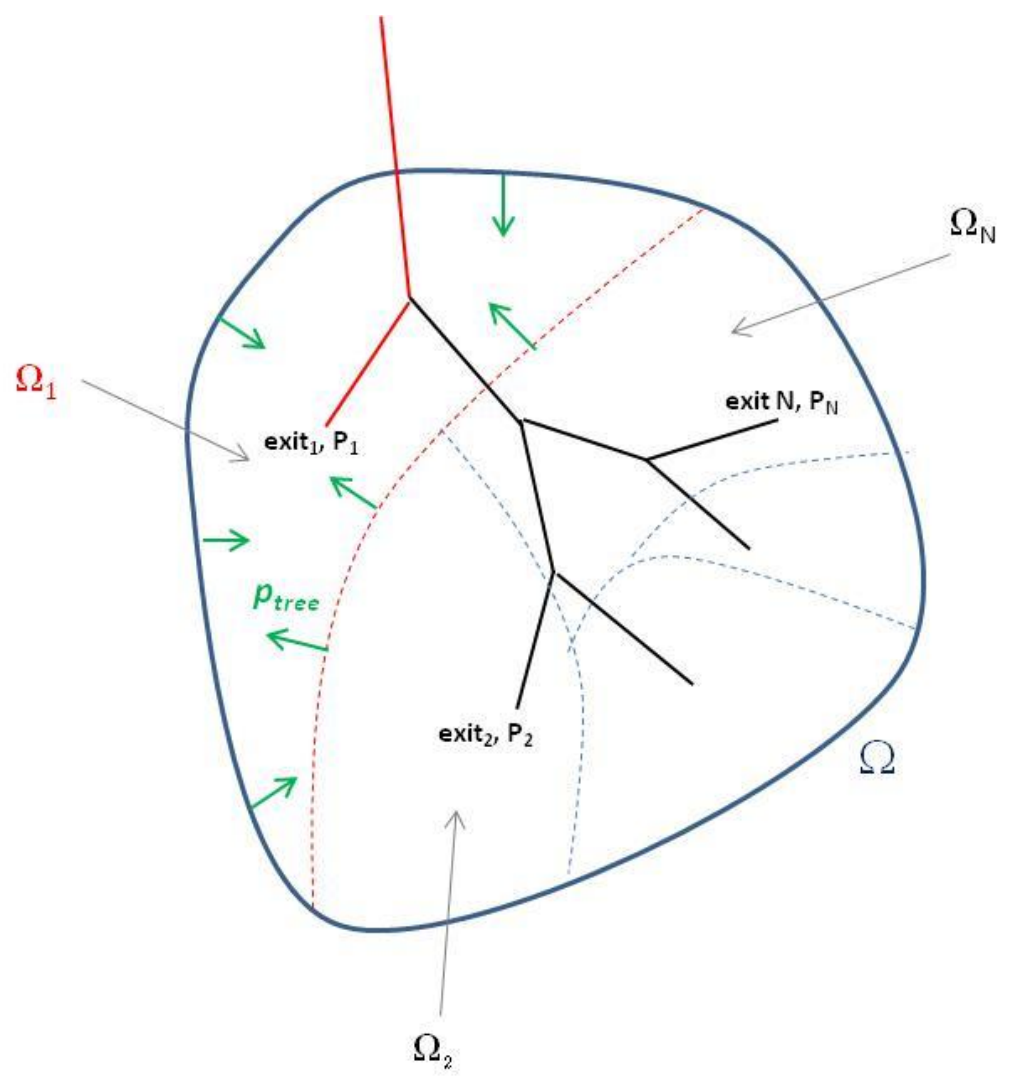

Figure 1: The parenchyma occupies a domain $\Omega$, fed in gas through a dyadic tree structure. This domain is subdivided into non-intersecting regions $\Omega_{i}$, each of them being supplied through the $i^{\text {th }}$ tree exit. Green arrows represent the apparent pressure applied on terminal region $\Omega_{1}$ due to the coupling with the tree. 

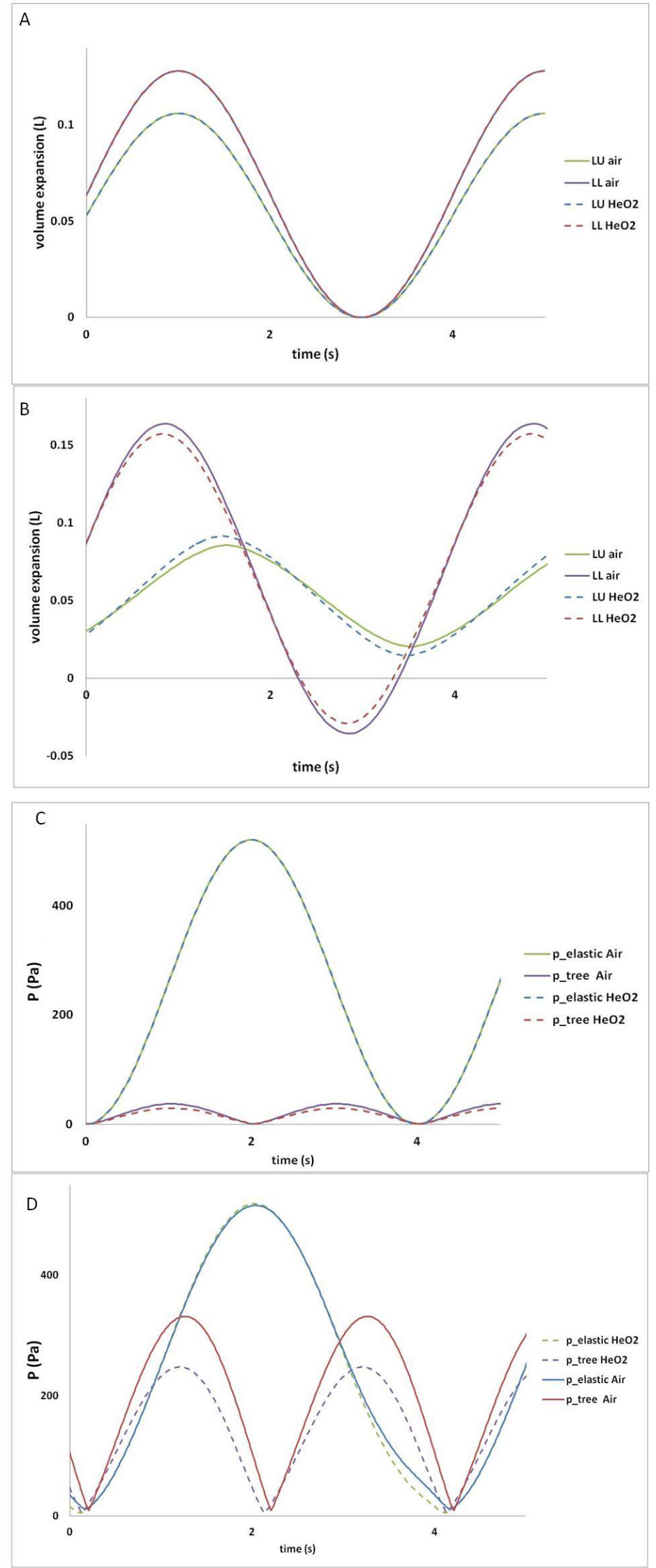

Figure 2: Lobar ventilation (A and B) and $P_{\text {elastic }}$ (eqn. (5)) and $\boldsymbol{P}_{\text {tree }}$ (eqn.(6)) efforts (C and D) through a sinusoidal respiration cycle for both air and $\mathrm{HeO}_{2}$ in a healthy situation (A and $C$ ) and when a bronchoconstriction is applied to LU branch (B: ratio 7, D: ratio 5). See the text for the ratio choices. 

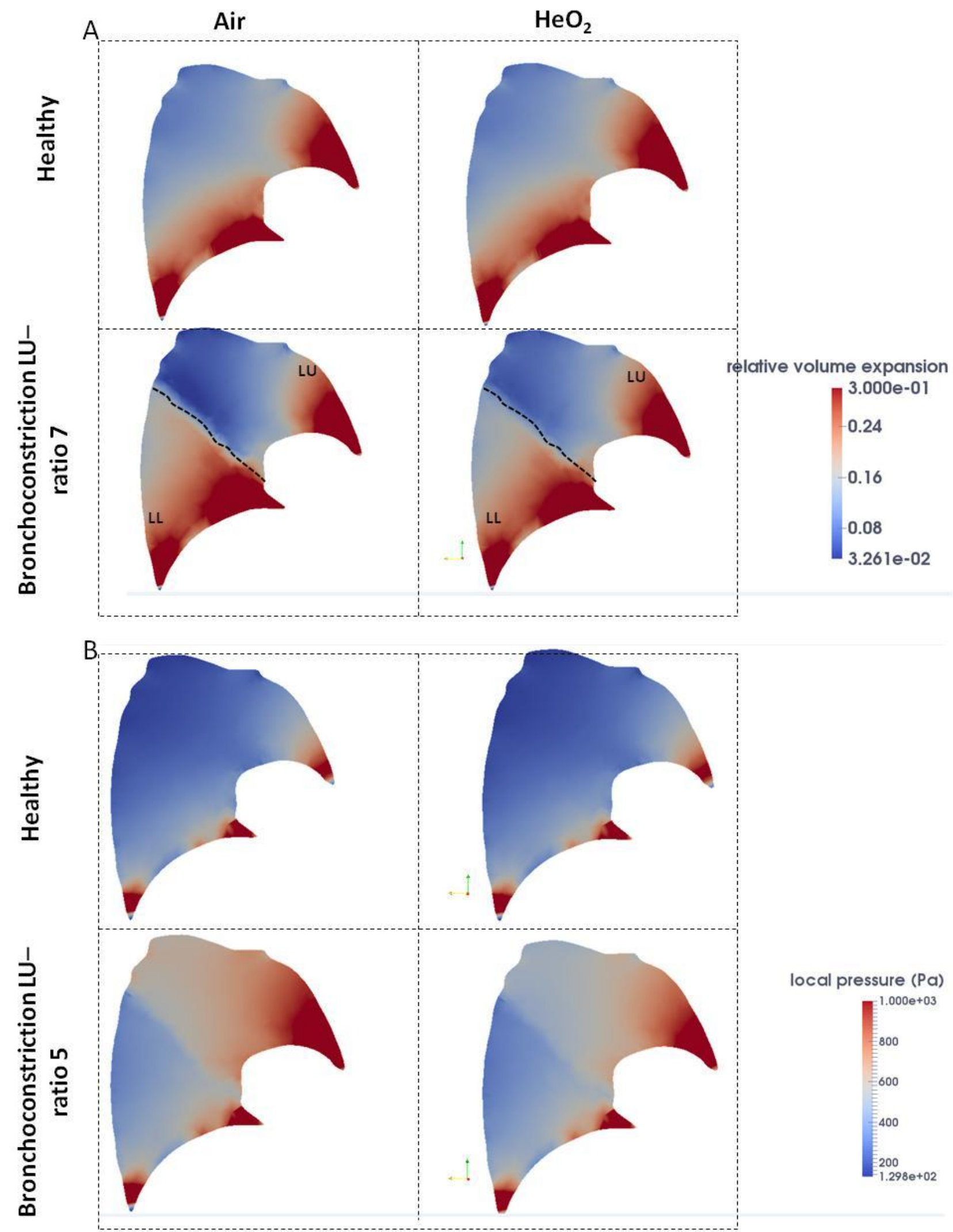

Figure 3: Left lung slice from basis to apex representing: $(A)$ the relative volume expansion $\operatorname{div}(u)$ in a healthy case and when a bronchoconstriction with ratio 7 is applied to $\mathrm{LU}$ branch, for both Air and $\mathrm{HeO}_{2}$ at time 1.68s, (B) the local pressure $p_{\text {eff }}$ defined by (4) in a healthy case and when a bronchoconstriction with ratio 5 is applied to $\mathrm{LU}$ branch, for both Air and $\mathrm{HeO}_{2}$ at time 1s. See the text for the ratio choices. 

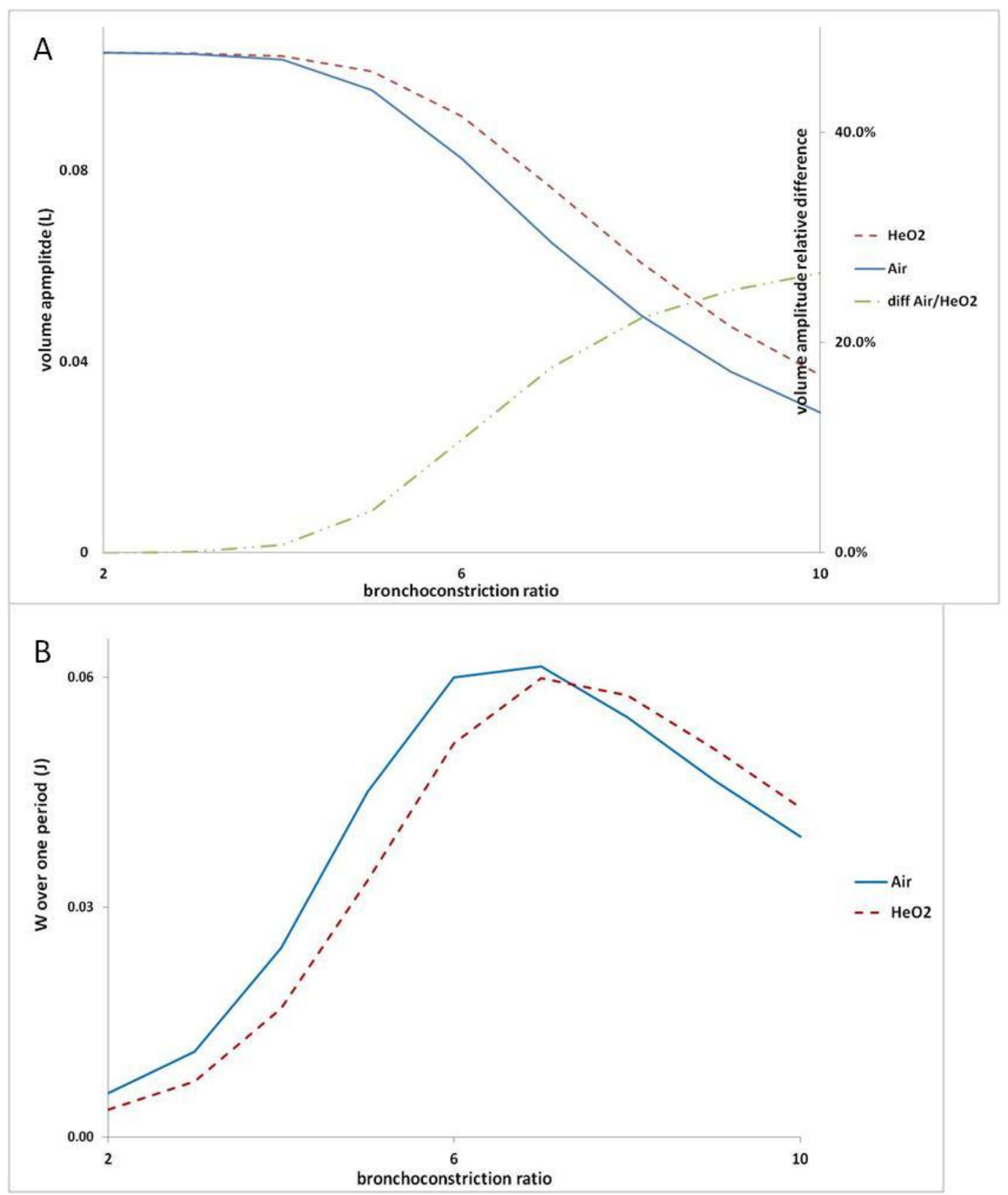

Figure 4: Bronchoconstriction applied on LU branch with varying ratios, evolution of: (A) LU lobe volume maximum amplitude over a breathing period for both $\mathrm{Air}$ and $\mathrm{HeO}_{2}$, and the relative difference between both gases, (B) $W$ (defined by eqn. (7)) over a breathing period for both $\mathrm{Air}$ and $\mathrm{HeO}_{2}$. 

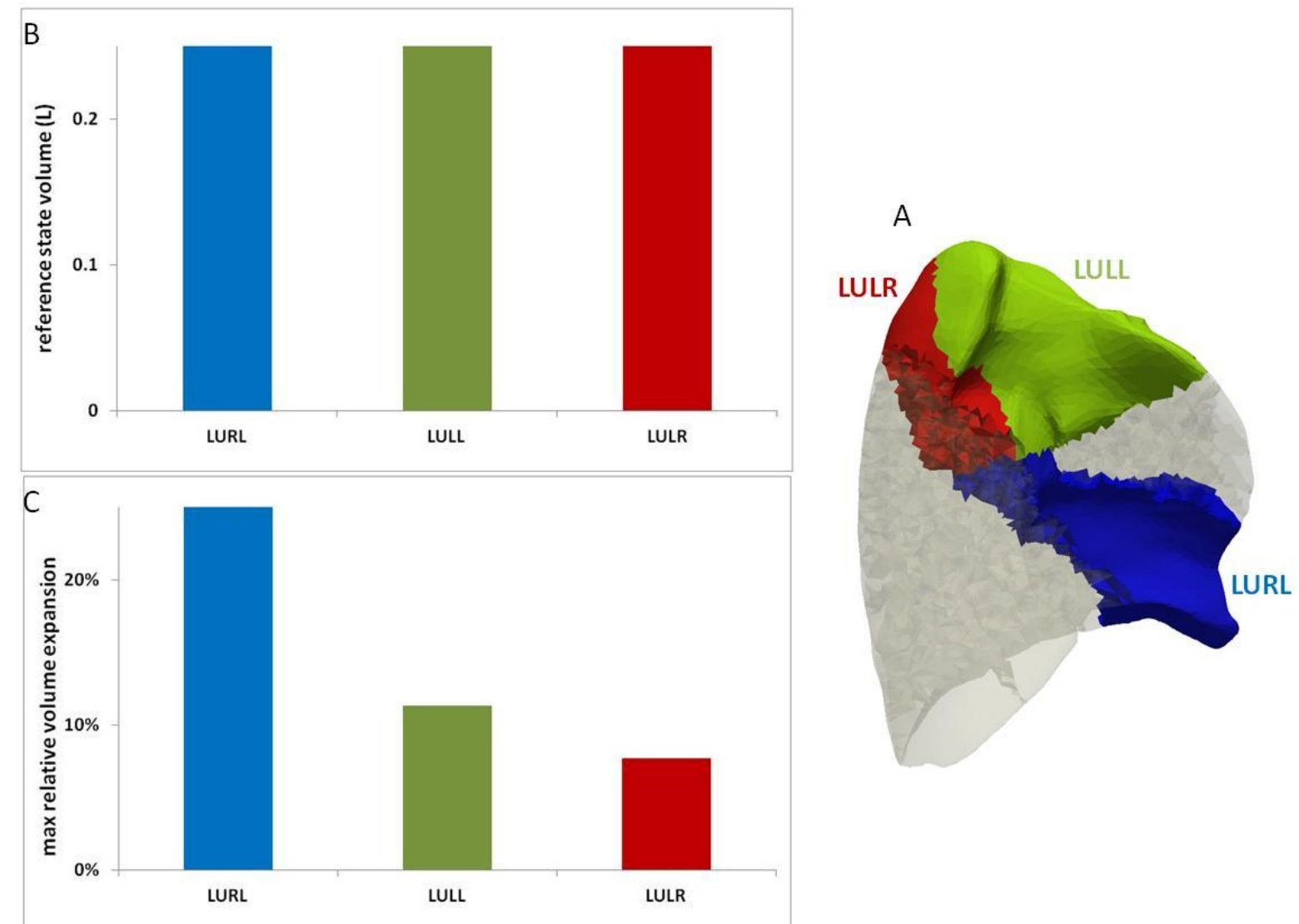

Figure 5: Three comparable regions belonging to the left lung are picked (A). They have the same reference state volume (B) but different volume expansions over the breathing cycle (C). 

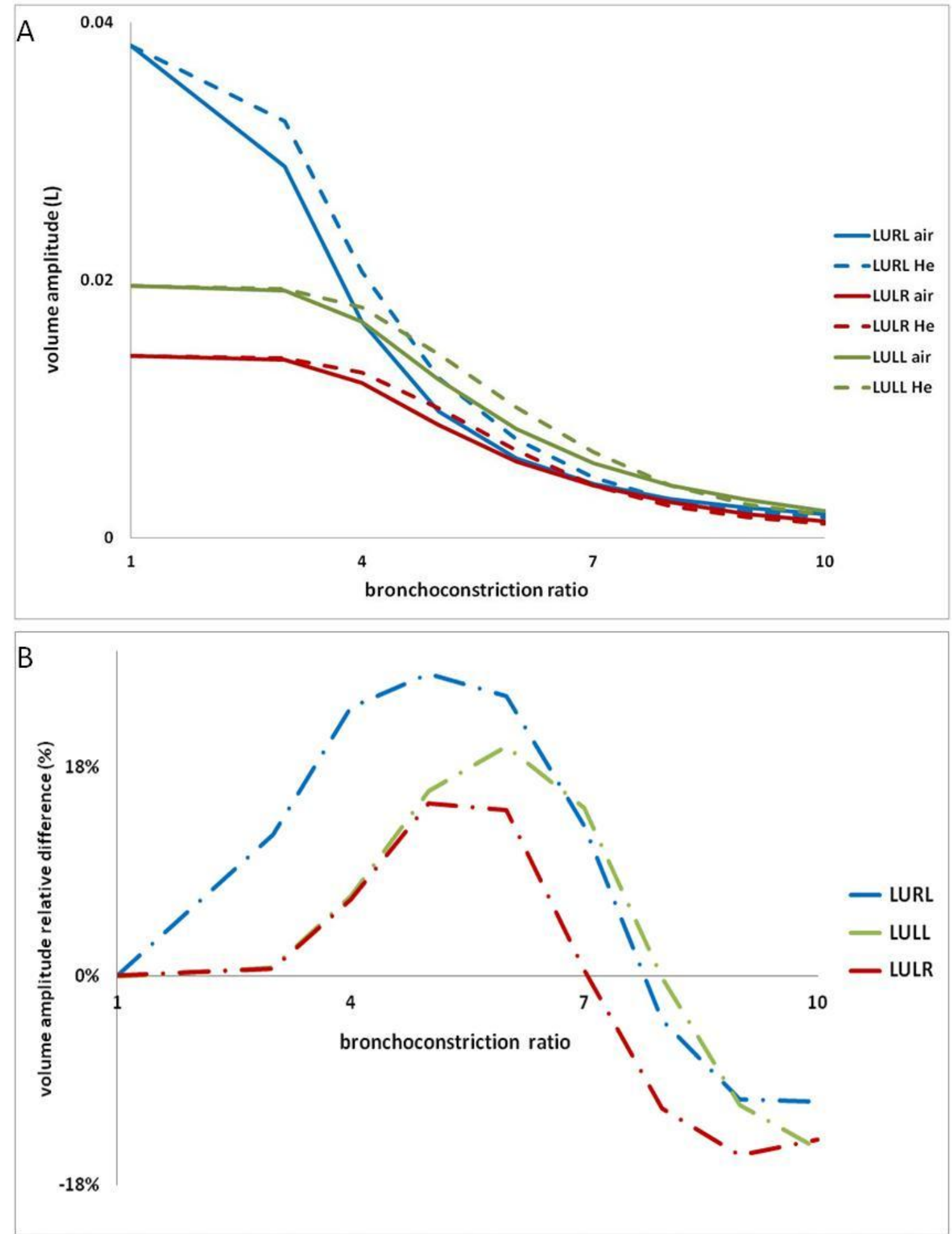

Figure 6: Ventilation in three lung regions. Volume amplitude with Air and $\mathrm{HeO}_{2}$ for varying ratios (A) and relative difference between both gases for each region (B). 


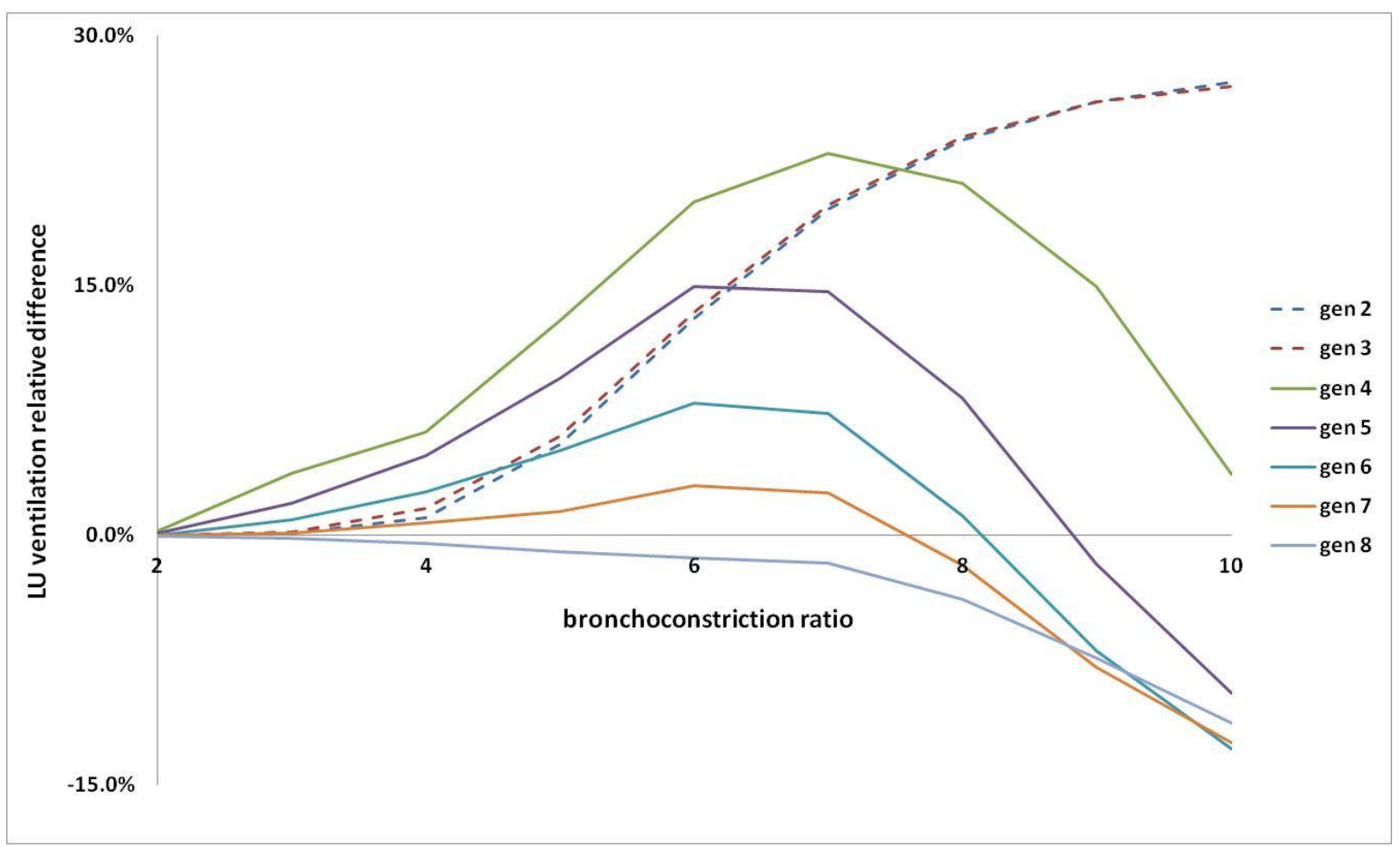

Figure 7: Bronchoconstrictions are applied to all the airways of a given generation belonging to LU lobe. Each curve corresponds to a different "diseased generation" and shows the maximum inhaled volume relative difference between both gases $\left(\frac{\operatorname{Vmax}_{\mathrm{HeO}_{2}}-\mathrm{Vmax}_{\mathrm{Air}}}{V \operatorname{Vmax}_{\text {Air }}}\right)$ for varying ratios. 


\begin{tabular}{|c|c|c|}
\hline $\begin{array}{l}\Delta \mathbf{V}_{\text {frc }}(\mathbf{L}) \text { Inhaled gas } \\
\mathbf{T}(\mathbf{s})-\text { tree model }\end{array}$ & Air & $\mathbf{H e O}_{\mathbf{2}}$ \\
\hline $0.23 \mathrm{~L} \& 4 \mathrm{~s}$ - healthy & 1 simulation & 1 simulation \\
\hline $0.16 \mathrm{~L} \& 2 \mathrm{~s}$ - asthma & 50 simulations & 50 simulations \\
\hline $0.23 \mathrm{~L} \& 4 \mathrm{~s}$ - asthma & 50 simulations & 50 simulations \\
\hline $0.23 \mathrm{~L} \& 8 \mathrm{~s}$ - asthma & 50 simulations & $\mathbb{3}$ \\
\hline $0.23 \mathrm{~L} \& 4 \mathrm{~s}$ - plug only & 50 simulations & 50 simulations \\
\hline $0.23 \mathrm{~L} \& 4 \mathrm{~s}-$ plug free & 50 simulations & 50 simulations \\
\hline
\end{tabular}

table 1: simulations performed on the asthma model. We generate fifty "asthma attack trees", for each we run breathing simulations with sinusoidal displacement boundary conditions, time period is $T$ and volume expansion along the cycle is $\Delta V_{\text {frc }}$. To simulate a crisis, we consider reduced $\Delta V_{\text {frc }}$ and $T$. To evaluate the benefit of slow breathing we take a longer period, this is done for air only since Heliox has little impact for low flows. An "asthmatic" tree contains both severe and moderate bronchoconstrictions as described in 2.4. Tree qualified as "plug only" and "plug free" respectively contain only severe bronchoconstrictions and only diffuse moderate bronchoconstrictions (see 2.4). "Healthy" means no bronchonconstrictions. Blue and red refer respectively to Figure 8 and Figure 9. 


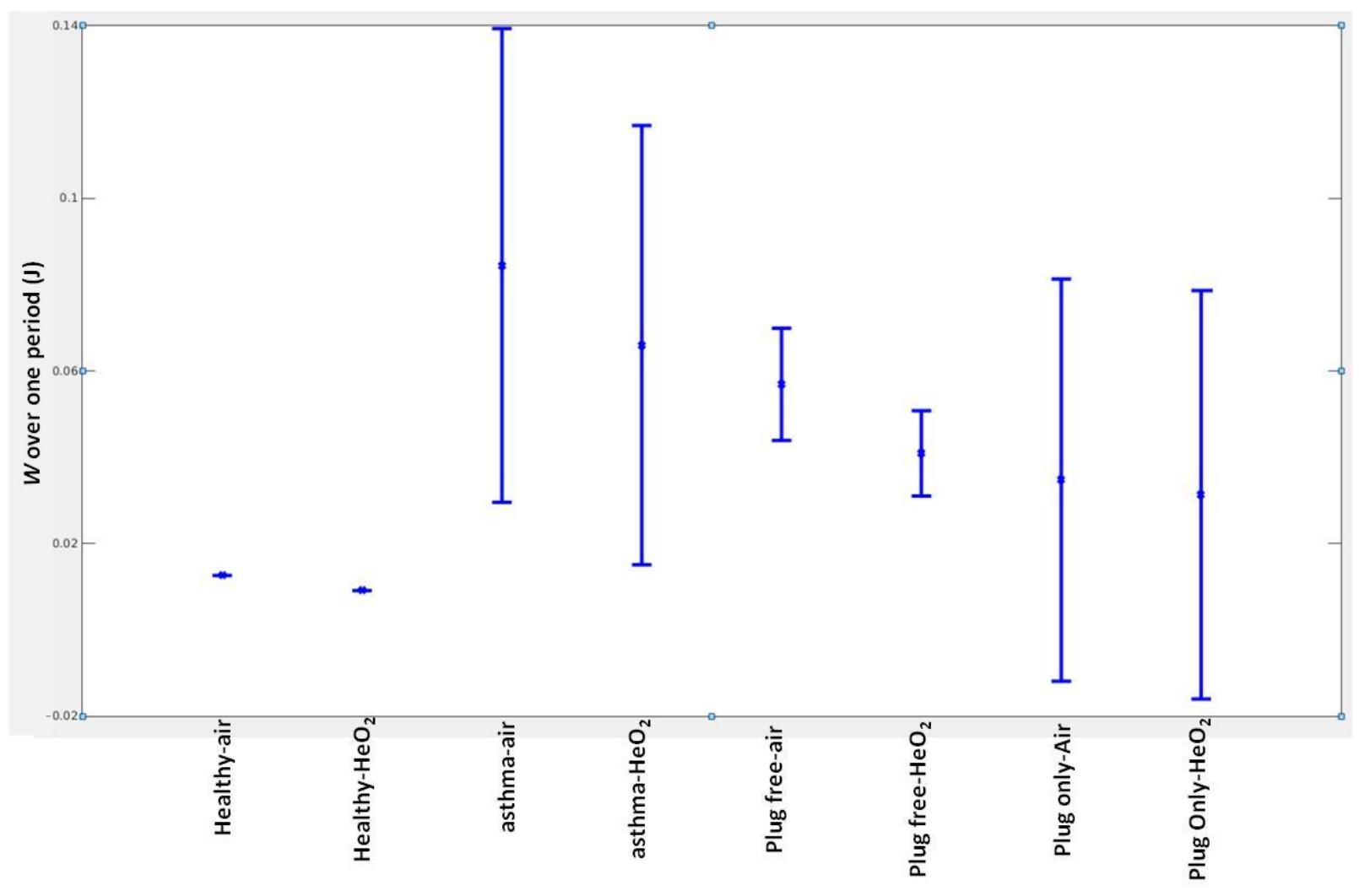

Figure 8: average and standard deviations of the $W$ over a respiration cycle in different configurations: one healthy patient breathing air and $\mathrm{HeO}_{2}$, fifty asthmatic configurations ith bronchonconstrictions simulated according to 2.4, fifty patients with only diffuse moderate bronchoconstrictions (plug free) and fifty patients with only severe bronchoconstrictions (plug only). 


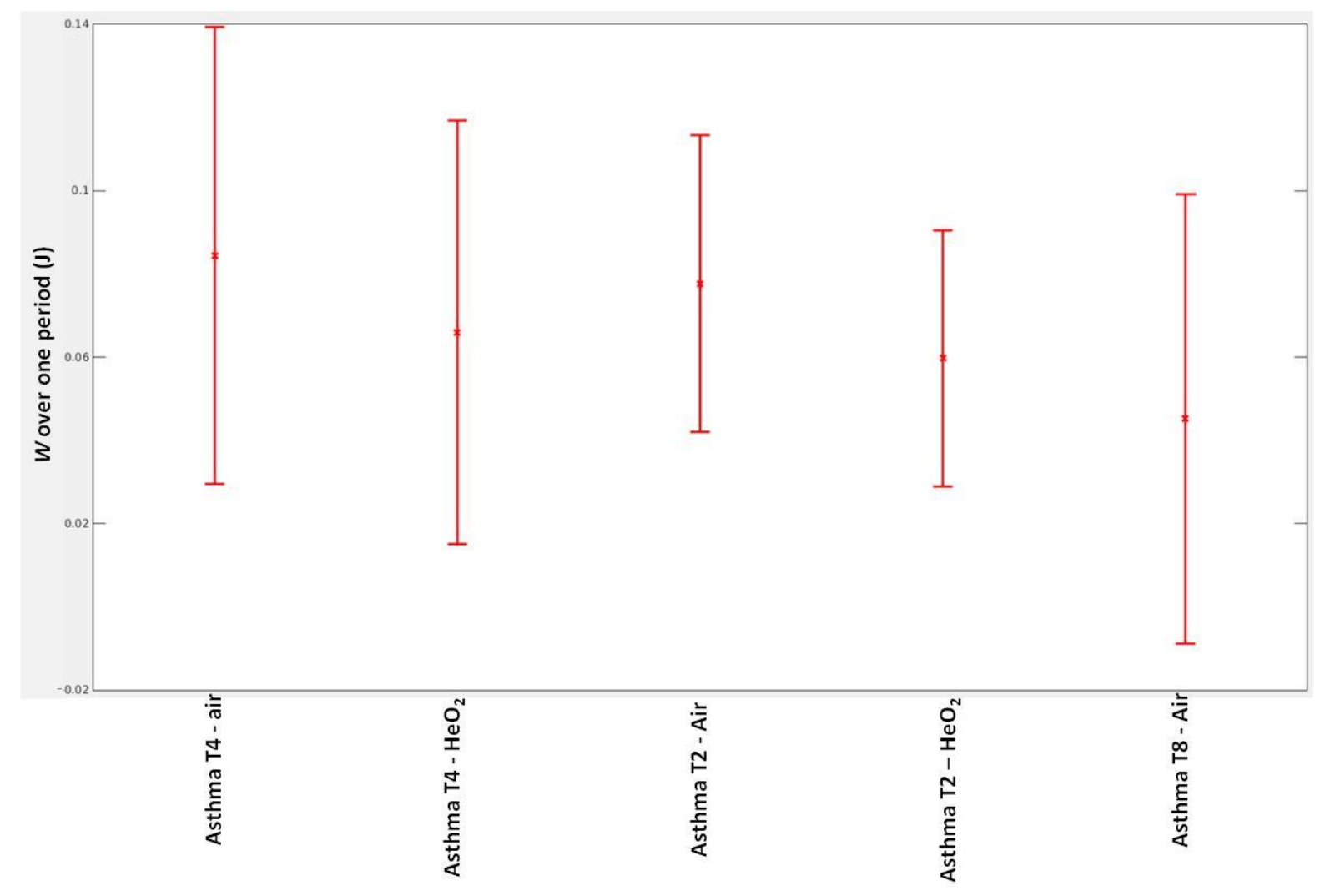

Figure 9: average and standard variations of the $W$ over a respiration cycle in different configurations: one healthy patient breathing air and $\mathrm{HeO}_{2}$, fifty asthmatic patients with bronchoconstrictions simulated according to 2.4 breathing air and $\mathrm{HeO}_{2}$ with time period $4 \mathrm{~s}$ and volume expansions $0.23 \mathrm{~L}$ (T4), the same fifty asthmatic patients breathing air and $\mathrm{HeO}_{2}$ with time period $2 \mathrm{~s}$ and volume expansions $0.16 \mathrm{~L}$ (T4), the same fifty asthmatic patients breathing air with time period $8 \mathrm{~s}$ and volume expansions $0.23 \mathrm{~L}$ (T8). 